\title{
Analgésicos, antipiréticos e anti-inflamatórios não esteroides em prescrições pediátricas
}

\author{
Analgesics, antipyretics and non-steroidal anti-inflammatory \\ drugs in pediatric prescriptions
}

Tânia Regina Ferreira ${ }^{1}$

Silvio Barberato Filho ${ }^{1}$

Adriano Ferreti Borgatto ${ }^{2}$

Luciane Cruz Lopes ${ }^{1}$

\footnotetext{
${ }^{1}$ Curso de Farmácia, Universidade de Sorocaba. Rodovia Raposo Tavares Km 92,5, Vila Artura. 18.023-000 Sorocaba SP Brasil.

taniasbragia@terra.com.br ${ }^{2}$ Departamento de Informática e Estatística, Centro Tecnológico, Universidade Federal de Santa Catarina.
}

\begin{abstract}
The use of analgesic, antipyretic and non-steroidal anti-inflammatory drugs by children more often than not neither have the approval of regulatory agencies nor the endorsement of scientific evidence. Pediatric prescriptions can be influenced by factors that do not promote the rational use of drugs by this category. The objective of this study was to evaluate the use of analgesic, antipyretic and non-steroidal anti-inflammatory drugs in children, considering the public (SUS) and private (N-SUS) sectors. The sample comprised 150 prescriptions (101 SUS and 49 NonSUS) followed by interviews with the caregivers in eighteen locations (nine private drugstores and nine units of the Unified Health System (SUS). The drugs were prescribed appropriately, by age group, only in $21.8 \%$ (SUS) and 29.6\% (NonSUS) prescriptions. Over $95 \%$ of prescription, regardless of source, did not meet the established criteria for evaluation of their rational use, with dosage, frequency and duration of treatment errors. The analysis of prescriptions for analgesic, antipyretic and non-steroidal anti-inflammatory drugs for children revealed no significant differences between the public and private sectors.

Key words Analgesics, Antipyretics, Non-steroidal anti-inflammatory drugs, Prescriptions, Use of medication, Pediatrics
\end{abstract}

Resumo O uso de analgésicos, antipiréticos e antiinflamatórios não esteroides por crianças muitas vezes não tem aprovação das agências reguladoras, nem respaldo das evidências científicas. Prescrições pediátricas podem ser influenciadas por fatores que não favorecem o uso racional dos medicamentos desta classe. O objetivo deste trabalho foi avaliar a utilização de analgésicos, antipiréticos e anti-inflamatórios não esteroides em crianças, considerando os setores público (SUS) e privado (N-SUS). A amostra foi composta por 150 prescrições (101 SUS e 49 N-SUS) seguidas de entrevista aos cuidadores, em dezoito locais (nove drogarias privadas e nove Unidades de Saúde do SUS). Os medicamentos foram prescritos de forma apropriada, segundo faixa etária, somente em $21,8 \%$ (SUS) e 29,6\% (N-SUS) das prescrições. Mais de 95\% das receitas, independente da origem, não atenderam aos critérios estabelecidos para avaliação do uso racional, com erros de dose, frequência e duração do tratamento. A análise das prescrições de analgésicos, antipiréticos e antiinflamatórios não esteroides para crianças não apresentou diferenças significantes nos setores público e privado.

Palavras-chave Analgésicos, Antipiréticos, Antiinflamatórios não esteroides, Prescrições, Uso de medicamentos, Pediatria 


\section{Introdução}

A prescrição pediátrica é direcionada ao acompanhante da criança, geralmente a mãe, que será responsável pela correta execução do tratamento, cujo sucesso depende óbvia e integralmente deste fato. Para que haja compreensão das prescrições, elas devem ser sempre escritas com letra legível, em linguagem compreensível, não devem conter símbolos e/ou abreviaturas e devem trazer explicações de todo seu conteúdo, adequado ao grau de entendimento do cuidador ${ }^{1}$. Alguns preceitos gerais sobre prescrição de medicamentos são obrigatórios e definidos por lei ${ }^{2,3}$, outros correspondem a Boas Práticas ${ }^{4}$ e ao Código de Ética Médica ${ }^{5}$.

Com frequência, a prescrição nas faixas pediátricas é baseada em extrapolações de doses e/ ou modificações de formulações para adultos, ignorando-se completamente as diferenças entre estes e crianças, e submetendo-as aos riscos de eficácia não comprovada ${ }^{6}$ e tomada de decisão duvidosa ${ }^{7}$. Por isso, no ato da prescrição, o profissional médico deve utilizar além de sua experiência clínica, as informações que adquire de diferentes fontes de informação baseadas em evidências científicas que favoreçam a seleção do melhor tratamento e sua adesão ${ }^{8}$, evitando-se preferencialmente o uso de medicamentos de introdução recente no mercado?.

Estima-se que $30 \%$ dos medicamentos prescritos para crianças pertençam ao grupo dos antiinflamatórios não esteroides ${ }^{10}$, apesar de sua to$x^{x i c i d a d e}{ }^{11}$ e de seus efeitos adversos ${ }^{12}$. Os analgésicos, antipiréticos (AA) e os anti-inflamatórios não esteroides (AINE) cuja indicação inclui o uso em pediatria, segundo o Formulário Terapêutico Nacional ${ }^{13}$, a Anvisa e a FDA, são: ácido acetilsalicílico, paracetamol, ibuprofeno e dipirona. Porém, mesmo sem ter seu uso em crianças aprovado pela Anvisa, fármacos como nimesulida, diclofenaco, naproxeno e piroxicam eram muito prescritos para uso em pediatria em algumas regiões do Brasil ${ }^{14}$. Não foram encontrados dados atualizados, que confirmem ou não o uso de tais medicamentos em crianças no Brasil.

Lista de medicamentos estaduais (Resme) e municipais (Remume) ainda incluem muitos destes fármacos cuja utilização não segue protocolos clínicos e recomendações baseadas em evidências clínicas ${ }^{15}$. O SUS poderia se beneficiar com pesquisas que pudessem identificar se o padrão de uso de medicamentos de elevado consumo, principalmente em população vulnerável (pediátrica), está relacionado ao tipo de profissi- onal prescritor vinculado a este e diverge do setor privado ou se este padrão é comum a ambos os setores. Tais achados levariam a intervenções distintas junto à classe médica e principalmente à gestão da Assistência Farmacêutica local. Além disso, a detecção pontual deste tipo de problema poderia favorecer o uso racional desta classe de medicamentos, o acesso daqueles considerados eficazes e seguros e identificar se os prescritores do SUS são influenciados por um padrão geral de prescrição ou se estão diretamente condicionados às listas de medicamentos locais. Assim, esta pesquisa se propôs avaliar de forma comparativa, o uso racional de analgésicos e antiinflamatórios não esteroides em crianças a partir de prescrições provenientes dos setores público (SUS - Sistema Único de Saúde) e privado (NSUS) na cidade de Sorocaba, São Paulo.

\section{Casuística e método}

Trata-se de estudo observacional, transversal, realizado a partir de análise de prescrições pediátricas e de informações fornecidas pelos cuidadores.

A coleta de dados ocorreu em nove farmácias privadas e em nove locais do setor público de Sorocaba, sendo estes: seis Unidades Básicas de Saúde (UBS), duas Unidades Pré-Hospitalares (UPH) e uma Clínica de Especialidades Médicas (Policlínica) por estarem inseridas em bairros populosos e por atenderem públicos com diferentes enfermidades e faixas de renda. A coleta de dados também ocorreu na Farmácia Comunitária Vital Brazil, que atua como farmácia-escola, sendo administrada pela Universidade de Sorocaba com apoio da PUC (SP) e atende gratuitamente um público bastante diversificado, incluindo prescrições provenientes de setor público e privado. A pesquisa foi iniciada após a aprovação do projeto pelo CEP - UNISO.

Foram consideradas as prescrições para crianças de 0 a 12 anos de idade, independente de etnia e sexo e entrevista aos cuidadores (responsáveis) maiores de 18 anos responsáveis diretamente pelos medicamentos, portando prescrição pediátrica contendo analgésico, antipiréticos e anti-inflamatórios não esteroides (AA e AINE), que se dispuseram a participar, mediante assinatura do Termo de Consentimento Livre e Esclarecido. Os voluntários, escolhidos para participar do estudo por meio de amostragem, por conveniência, não probabilística, tiveram a receita solicitada para que dela fossem copiados os dados necessários sendo imediatamente devolvida. 
Em seguida foram convidados a responder um questionário composto por perguntas estruturadas, fechadas e abertas que contemplaram variáveis relacionadas ao paciente, à prescrição de AA e AINE, ao tratamento e dados relativos ao cuidador.

\section{Análise dos dados}

Para avaliação do uso racional levou-se em conta se o medicamento era apropriado (para a idade e se havia riscos de interações graves ou de contraindicações), se dose, frequência e duração estavam adequadas. Foi considerado inadequado a ausência de dados e informações incompletas e que poderiam causar dúvidas e/ou induzir a erros de utilização. Para o referencial teórico seguiram-se as informações contidas no Drugdex ${ }^{12}$, além disso, para a verificação da adequação da dose, da frequência e da duração do tratamento utilizaram-se os dados provenientes das respectivas bulas e informações fornecidas pelo fabricante do medicamento.

Foram verificadas possíveis associações entre SUS e N-SUS com as diferentes variáveis. Para análise da relação existente entre as variáveis, aplicou-se o teste Qui-Quadrado e em algumas o teste Exato de Fisher. A força da associação entre o desfecho e as variáveis independentes foi estimada através do cálculo de razões de prevalência e seus respectivos intervalos de confiança de 95\%.

\section{Resultados}

Os dados do estudo foram coletados nos meses de janeiro a setembro de 2009, em diferentes dias, contemplando os quatro períodos sazonais. Foram colhidas informações referentes a 150 receitas e entrevistas aos cuidadores nos diversos locais selecionados. Destas, 101 são provenientes do SUS e 49 do N-SUS. Este último incluiu receitas provenientes de convênios, cooperativas e particulares. A idade dos pacientes variou de cinco dias a 12 anos, com mediana de 4,8 anos. Foram 431 medicamentos prescritos, sendo 295 contidos em prescrições do SUS e 136 N-SUS. Este total corresponde a 68 fármacos, divididos em 35 classes terapêuticas (ATC), com a média de 2,9 medicamentos por paciente atendido no SUS e 2,8 por pacientes N-SUS. Nas receitas do SUS, em $88,1 \%$ estavam prescritos medicamentos constantes na Rename 2008, enquanto no NSUS apenas $68,5 \%, \mathrm{p}<0,001$. Tanto nas receitas provenientes do SUS $(64,3 \%)$ quanto N-SUS
(51,0\%) verifica-se prevalência de três ou mais medicamentos. Mais pacientes do N-SUS $(51,0 \%)$ usaram medicamentos não incluídos nas prescrições que os pacientes SUS (32,6\%), $\mathrm{p} \leq 0,05$. Constatou-se a maior prevalência de receitas prescritas por pediatras, mas destaca-se que em mais da metade das prescrições não consta a especialidade do médico. Quanto ao grau de escolaridade do cuidador pode-se observar que $17,8 \%$ SUS e $14,8 \%$ N-SUS tinham menos de três anos de estudo. O cuidador da criança entrevistado no SUS foi, em sua maioria, mães $(53,4 \%)$ diferentemente do setor privado onde o cuidador era o avô/avó (30,6\%), $(\mathrm{p} \leq 0,05)$ Tabela 1 .

Quase a totalidade das receitas, independente da origem, não apresenta orientações não medicamentosas. Em mais de 79,8\% das receitas não foi encontrada a duração do tratamento, elemento que além de auxiliar o próprio usuário, é importante para que outros profissionais da saúde possam orientar a utilização dos medicamentos prescritos. Em 92,6\% do total de receitas não continham a idade dos pacientes e em $96,6 \%$ não constavam o peso corporal. Quando se comparam estas duas variáveis (idade e peso) com a procedência da receita, verifica-se que nas do SUS $(97,1 \%$ e $99,0 \%)$ a ausência destes dados é maior que nas do N-SUS $(83,7 \%$ e $91,9 \%),(p \leq 0,05)$.

Tanto SUS (110 medicamentos) quanto NSUS (54 medicamentos) continham mais de um AA e AINE prescrito por receita. Foram observadas diferenças estatísticas significantes $(\mathrm{p} \leq 0,05)$, para a indicação da dipirona $(61,8 \%)$ no SUS e do cetoprofeno no N-SUS (18,5\%). Dois AA e AINE se destacam no SUS, dipirona e ibuprofeno pela prevalência de prescrição. Já no setor privado destacam-se ibuprofeno, dipirona, cetoprofeno e paracetamol nesta ordem de prevalência (Tabela 2).

Constatou-se $12,9 \%$ SUS e $12,2 \%$ N-SUS de interações medicamentosas contraindicadas, isto é, aquelas que impedem absolutamente a continuação do uso. Segundo British National Formulary ${ }^{16}$, ibuprofeno reduz a excreção de metotrexato e de seu metabólito, aumentando o risco de toxicidade, principalmente em crianças. Esta interação é classificada como grave, isto é, aquela que pode por em risco a vida do paciente e requer intervenção médica para prevenir ou minimizar os efeitos adversos. Uma prescrição do SUS continha esta associação de medicamentos (Tabela 3).

Os sete fármacos AA e AINE contidos nesta amostra foram prescritos 164 vezes (SUS e NSUS), nas 150 receitas analisadas. Ambos os setores prescreveram o medicamento apropriado 
Tabela 1. Caracterização das prescrições pediátricas $(n=150)$ contendo analgésicos, antipiréticos e anti-inflamatórios não esteroides, provenientes da rede pública (SUS) e privada (N-SUS) de Sorocaba (SP), jan./set. 2009.

\begin{tabular}{|c|c|c|c|c|c|}
\hline \multirow{2}{*}{ Variável } & \multicolumn{2}{|c|}{$\begin{array}{l}\text { Setor público } \\
\quad(n=101)\end{array}$} & \multicolumn{2}{|c|}{$\begin{array}{l}\text { Setor privado } \\
\quad(\mathrm{n}=49) \\
\end{array}$} & \multirow{2}{*}{ p-valor } \\
\hline & $\mathbf{n}$ & $\%$ & n & $\%$ & \\
\hline Especialidade Médica & 28 & 27,7 & 20 & 40,8 & $0,263^{* *}$ \\
\hline Pediatria & 9 & 8,9 & 3 & 6,1 & \\
\hline Outra especialidade & 64 & 63,3 & 26 & 53,0 & \\
\hline \multicolumn{6}{|l|}{ Não consta } \\
\hline Faixa etária * & 17 & 16,3 & 4 & 8,1 & $0,116^{* *}$ \\
\hline Menos de 1 ano & 37 & 36,6 & 21 & 42,8 & \\
\hline De 1 até 4 anos & 27 & 26,6 & 8 & 16,3 & \\
\hline Mais de 4 até 7 anos & 20 & 19,8 & 16 & 32,6 & \\
\hline \multicolumn{6}{|l|}{ Mais de 7 anos } \\
\hline Quantidade medicamentos na receita & 10 & 9,9 & 8 & 16,3 & $0,262^{* *}$ \\
\hline 1 & 26 & 25,7 & 16 & 32,6 & \\
\hline 2 & 65 & 64,3 & 25 & 51,0 & \\
\hline \multicolumn{6}{|l|}{$\geq 3$} \\
\hline Uso de outros medicamentos não incluídos na receita & 33 & 32,6 & 25 & 51,0 & $0,031^{* *}$ \\
\hline
\end{tabular}

*Formulário Terapêutico Nacional, 2010, modificado. ${ }^{* *}$ p-valor para teste Qui-Quadrado.

Tabela 2. AA e AINE presentes nas receitas pediátricas atendidas por drogarias e farmácias da rede pública e privada de Sorocaba, SP, jan./set. 2009.

\begin{tabular}{|c|c|c|c|c|c|c|}
\hline \multirow[b]{2}{*}{ Nome do fármaco } & \multirow[b]{2}{*}{ ATC $^{*}$} & \multicolumn{2}{|c|}{ SUS $(n=101)$} & \multicolumn{2}{|c|}{ N-SUS $(n=49)$} & \multirow[b]{2}{*}{$\mathrm{p}^{* *}$} \\
\hline & & $\mathrm{n}$ & $\%$ & $\mathrm{n}$ & $\%$ & \\
\hline ácido acetilsalicílico & N02BA01(4), B01A (1) & 5 & 4,5 & 0 & 0 & $0,173^{x}$ \\
\hline cetoprofeno & M01AE03 & 6 & 5,4 & 10 & 18,5 & $0,038^{\star}$ \\
\hline dipirona & N02BB02 & 68 & 61,8 & 14 & 25,9 & $<0,001^{* * x}$ \\
\hline ibuprofeno & M01AE01 & 22 & 20,0 & 18 & 33,3 & $0,081^{* * *}$ \\
\hline nimesulida & M01AX17 & 0 & 0 & 2 & 3,7 & $0,105^{\star}$ \\
\hline paracetamol & N02BE01 & 9 & 8,2 & 8 & 14,8 & $0,285^{* * x}$ \\
\hline piroxicam & M01AC01 & 0 & 0 & 2 & 3,7 & $0,105^{\star}$ \\
\hline Total & & 110 & 100 & 54 & 100 & \\
\hline
\end{tabular}

"ATC Anatomical Therapeutic Chemical. "p-valor do teste exato de Fisher. ${ }^{* * *}$ p-valor para teste Qui-Quadrado.

em aproximadamente $70 \%$ das vezes, mas se equivocam na dose, frequência e duração, pois somente $13,6 \%$ (SUS) e $24,0 \%$ (N-SUS) o fazem na dose adequada; $12,7 \%$ (SUS) e 24,0 \% (N-SUS) foram feitas com frequência correta e, surpreendentemente, $2,7 \%$ (SUS) e $0 \%$ (N-SUS) com a duração adequada, Tabela 4.

\section{Discussão}

Considerando o número de farmácias e drogarias privadas de Sorocaba (170), inicialmente o tra- balho se propôs a entrevistar os pacientes em $10 \%$ destes locais. No entanto, houve dificuldade em se obter autorização para a permanência da pesquisadora nestes estabelecimentos privados e por isso a coleta se deu em nove locais, distribuídos em cinco macrorregiões do município. Ressalta-se que o tamanho da amostra ou o método utilizado na coleta dos dados podem ser responsáveis pelas situações em que não foram observadas associações estatísticas.

O índice médio do número de medicamentos prescritos por receita $(2,98)$ foi um pouco superior ao de outros estudos voltados à prescrição 
Tabela 3. Caracterização das interações medicamentosas quanto aos AA e AINE prescritos e à severidade, em receitas pediátricas provenientes da rede pública (SUS) e privada (N-SUS) de Sorocaba-SP, jan./set. 2009.

\begin{tabular}{|c|c|c|c|c|c|c|}
\hline \multirow[b]{2}{*}{ Fármacos } & \multirow[b]{2}{*}{ Associações } & \multicolumn{2}{|c|}{ SUS $(n=101)$} & \multicolumn{2}{|c|}{ N-SUS $(n=49)$} & \multirow[b]{2}{*}{ p-valor } \\
\hline & & $\mathbf{n}$ & $\%$ & $\mathbf{n}$ & $\%$ & \\
\hline \multicolumn{7}{|c|}{ Interações contraindicadas } \\
\hline \multirow[t]{5}{*}{ dipirona } & + ácido acetilsalicílico & 5 & 4,9 & 0 & 0 & $0,173^{*}$ \\
\hline & + cetoprofeno & 3 & 3,0 & 1 & 2,0 & $1,000^{*}$ \\
\hline & + ibuprofeno & 3 & 3,0 & 1 & 2,0 & $1,000^{*}$ \\
\hline & + nimesulida & 0 & 0 & 2 & 4,0 & $0,105^{*}$ \\
\hline & + paracetamol & 0 & 0 & 1 & 2,0 & $0,327^{*}$ \\
\hline \multirow[t]{2}{*}{ ibuprofeno } & + cetoprofeno & 1 & 1,0 & 1 & 2,0 & $0,548^{*}$ \\
\hline & + paracetamol & 1 & 1,0 & 0 & 0 & $1,000^{*}$ \\
\hline Subtotal & & 13 & 12,9 & 6 & 12,2 & \\
\hline \multicolumn{7}{|c|}{ Interações graves } \\
\hline ibuprofeno & + metotrexato & 1 & 1,0 & 0 & 0 & $1,000^{*}$ \\
\hline
\end{tabular}

${ }^{*}$-valor do teste exato de Fisher.

Tabela 4. Conformidade das prescrições pediátricas do SUS e N-SUS contendo AA e AINE segundo os indicadores para o uso racional de medicamentos, Sorocaba-SP, jan./set. 2009.

\begin{tabular}{|c|c|c|c|c|c|}
\hline \multirow[b]{2}{*}{ Indicadores de uso racional } & \multicolumn{2}{|l|}{ SUS } & \multicolumn{2}{|c|}{ N-SUS } & \multirow[b]{2}{*}{ p-valor } \\
\hline & $\mathrm{n}=110$ & $\%$ & $\mathrm{n}=54$ & $\%$ & \\
\hline Medicamento apropriado & 86 & 78,2 & 38 & 70,4 & 0,500 \\
\hline Dose certa & 15 & 13,6 & 13 & 24,0 & $0,046^{*}$ \\
\hline Frequência certa & 14 & 12,7 & 13 & 24,0 & $0,049^{*}$ \\
\hline Duração certa & 3 & 2,7 & 0 & 0 & 0,551 \\
\hline
\end{tabular}

"p-valor do Teste Qui-Quadrado seguido de Teste exato de Fisher. Medicamento apropriado = adequado para as características dos pacientes (idade) e que não apresentava riscos de interação grave ou contraindicação.

pediátrica como, por exemplo, em Ribeirão Preto (SP) $(2,2)$, em Araraquara (SP) $(2,5)$; em Tabatinga (SP) $(2,6)$; em Campo Grande (MS) $(2,3)^{14,17-19}$.

Não foram encontradas diferenças significantes entre o índice médio de medicamentos prescritos no SUS $(3,0)$ e no setor privado $(2,8)$. Se considerarmos que $30 \%$ dos cuidadores $(n=58)$ informaram que as crianças estavam utilizando medicamentos não prescritos na receita atual, correspondendo a 75 medicamentos a mais do que o total já contabilizado, este dado eleva o índice de consumo médio de medicamento por criança de 2,98 para 3,3, independente da origem das receitas. Pode-se notar que, em países onde existe controle menos rígido sobre a propaganda e comercialização de medicamentos, o número de fármacos prescritos por consulta chega ser duas a três vezes maiores do que aquele observado em países nórdicos ${ }^{20,21}$.

Somente $12 \%$ das receitas continham um medicamento e $60 \%$ destas continham mais de três. Este indicador reflete o nível de polimedicação encontrado nestas receitas. Levando-se em conta que a mediana da idade foi de 4,8 anos, que a faixa etária prevalente foi de 1 a 4 anos $(38,6 \%)$, nota-se que as prescrições contendo maior número de medicamentos ocorreram em faixas etárias menores, por exemplo, em crianças com menos de um ano $(\mathrm{n}=21)$ cerca de $80 \%$ $(\mathrm{n}=17)$ tinham prescrição de três ou mais medicamentos, comparado às crianças com mais de sete anos de idade ( $\mathrm{n}=36)$ com $47,2 \%(\mathrm{n}=17)$ das prescrições. Estes resultados são similares a outros dados de literatura onde os autores referem maior consumo de medicamentos nos dois 
primeiros anos de vida ${ }^{22,23}$. De qualquer forma, a prevalência de utilização de mais de três medicamentos nesta amostra foi muito superior ao encontrado em Ribeirão Preto (SP) (32,1\%) e foi similar ao encontrado em Tabatinga (SP) (50\%), demonstrando a intensa medicalização das crianças desde o início de suas vidas.

As pessoas acreditam que encontrarão a solução para todos os males nos medicamentos. Cada vez mais se estimula a medicalização da vida, com consequente aumento do uso de medicamentos ${ }^{24}$. Por outro lado, o consumo de medicamentos é, também, um indicador importante para avaliar a qualidade dos serviços de saúde e a efetividade da propaganda de medicamentos junto aos médicos e à população ${ }^{25}$. Pacientes do setor privado $(51,0 \%)$ usam mais medicamentos não incluídos nas prescrições que os pacientes SUS (32,6\%), $\mathrm{p} \leq 0,05$. Estes achados preocupam quando se trata de interação medicamentosa grave ou contraindicada, gerando riscos desnecessários, principalmente nesta faixa etária. A interação medicamentosa contraindicada nesta amostra ocorreu entre dois antipiréticos, confirmando os estudos realizados na Argentina ${ }^{26}$ e na Venezuela ${ }^{27}$ onde os clínicos prescrevem os antipiréticos para serem utilizados de maneira alternada. Trata-se, sem dúvida, de uso não apropriado.

Resultado bastante interessante deste trabalho é que nas receitas do SUS, em $88,1 \%$ estavam prescritos medicamentos constantes na Rename 2010 , enquanto no setor privado apenas $68,5 \%, \mathrm{p}$ $<0,001$. Outros estudos encontraram valores inferiores a este com relação ao SUS, por exemplo, Ribeirão Preto (SP) teve 82,5\% de concordância, $47 \%$ em Araraquara (SP), 32,6\% em Tabatinga (SP) e apenas em Campo Grande (MS) esse valor foi ligeiramente superior com $89,5 \%^{14,18,19,28}$.

Os AINE estão entre os fármacos mais frequentemente utilizados em todo o mundo. Nos Estados Unidos, eles respondem por mais de 70 milhões de prescrições e mais de 30 bilhões de comprimidos de venda livre comercializados anualmente ${ }^{29}$. Além disso, vários estudos mostram que os AA e os AINE estão entre os medicamentos mais utilizados por crianças, com ou sem prescrição médica' ${ }^{10,14,30-32}$ no Brasil. Em vários países desenvolvidos a indicação da faixa etária para uso deste grupo é bastante restrita, devido aos poucos estudos do seu uso em crianças abaixo de 12 anos ou mesmo em adolescentes. Nesta amostra estes fármacos foram o terceiro grupo mais prescrito. Os sete fármacos encontrados foram prescritos 164 vezes, sendo 110 em recei- tas do SUS e 54 do setor privado. Este total equivale a 1,09 medicamentos desta classe por paciente atendido. No SUS prevaleceram prescrições de dipirona (61,8\%) e ibuprofeno (20,0\%), $\mathrm{p} \leq 0,05$. Já no setor privado, foram mais frequentes as prescrições com: ibuprofeno $(33,3 \%)$, dipirona $(25,9 \%)$, cetoprofeno $(16,7 \%)$ e paracetamol $(14,8 \%)$, resultados idênticos ao encontrado por Moraes et al. ${ }^{33}$.

Ácido acetilsalicílico (ASA), ibuprofeno e paracetamol são os agentes mais utilizados para a redução da febre em crianças. Ao longo dos últimos 20 anos, por causa da associação entre o uso de ASA e a síndrome de Reye, a frequência de uso do ibuprofeno e do paracetamol vem aumentando ${ }^{34}$.

Síndrome de Reye é uma condição caracterizada por encefalopatia não inflamatória aguda $\mathrm{e}$ insuficiência hepática. A associação desta síndrome com o uso de salicilatos foi demonstrada em vários estudos epidemiológicos de todo o mundo. No entanto, menos de $0,1 \%$ das crianças que tomaram ASA desenvolveram a síndrome, mas, $80 \%$ dos pacientes diagnosticados com a síndrome de Reye tinham tomado ASA nas últimas 3 semanas $^{35,36}$. O nexo causal foi questionado com base em preconceitos e limitações nos estudos, mas as recomendações das agências governamentais de saúde que as crianças não sejam tratadas com salicilatos levaram a uma diminuição imediata e dramática na incidência da síndrome de Reye ${ }^{34,35}$.

Além disso, ASA é contraindicado para crianças menores de seis anos, devido a sua maior toxicidade em comparação com outros antipiréti$\cos ^{11,37}$.

Em virtude das eficácias antipiréticas comparáveis e perfis de tolerabilidade favoráveis, ibuprofeno e paracetamol, quando utilizados de forma correta, com formulações adequadas para a faixa etária, devem continuar a ser a terapia de primeira linha para o tratamento da febre infanti1 ${ }^{33,38,39}$.

As prescrições SUS continham frequentemente mais dipirona do que outros analgésicos e antipiréticos. Isso pode ser explicado pela presença deste medicamento na relação daqueles fornecidos pelo município. No entanto, o uso deste fármaco está permeado por muita controvérsia devido especialmente a associação com o aparecimento de mielotoxicidade proibindo seu uso em muitos países na década de 70. Mas muitos países, como por exemplo, Espanha, México, Índia, Egito, Brasil, Polônia, Rússia, Turquia, Bulgária e Alemanha, a dipirona, ainda é comercializada amplamente como medicamento isento de pres- 
crição médica, pois a incidência de agranulocitose nesses países é casuística ${ }^{40,41}$. As análises recentes mostram que os resultados sobre a incidência de mielotoxicidade e uso de dipirona podem estar superestimados. O risco estimado desta complicação mostra uma variabilidade geográfica elevada, o que é susceptível de refletir diferenças genéticas das populações avaliadas em estudos epidemiológicos. Hedenmalm e Spigset ${ }^{42}$ relataram a incidência de 1 caso por 1.431 prescrições na Suécia. Ibanez et al. ${ }^{43}$ encontraram que, na Espanha, o risco de agranulocitose quando do uso de dipirona em doses habituais e por um curto período de tempo é extremamente baixo, ou seja, 0,56 casos/habitante/ano. Entretanto, também observaram que o risco de agranulocitose fatal aumenta em vinte vezes com o prolongamento da administração ${ }^{43,44}$.

A única recomendação acerca do uso do medicamento encontrada nas prescrições de ambos os setores $(72,6 \%)$ foi: "usar se febre e/ou dor". Esta expressão é entendida como: a avaliação do uso do medicamento fica a cargo do responsável pela criança e não mais do clínico. Como se isso não fosse o bastante para que erros ocorressem, o prescritor não informa qual o máximo de dose que pode ser administrado em um dia e qual o intervalo mínimo entre uma dose e outra. Estão os responsáveis cientes da dose máxima e do intervalo de uso do medicamento? Sabem eles os riscos aos quais se podem expor uma criança utilizando um AA ou AINE em dose ou esquema posológico inadequado? Este panorama atual mostra, em parte, a falta de compromisso do prescritor com o paciente depois que o mesmo deixa o consultório.

Os pais costumam ter falsos temores e crenças sobre a febre, superestimando seus possíveis perigos; fato conhecido como Fobia da Febre. O medo infundado leva ao tratamento desnecessário da febre e ao uso excessivo dos serviços de saúde ${ }^{45,46}$.

Usualmente, define-se como febre a temperatura retal igual ou superior a $38^{\circ} \mathrm{C}$ ou temperatura axilar acima de $37,3^{\circ} \mathrm{C}^{1}$. Para um recémnascido, definiu-se febre como uma temperatura retal $>38^{\circ} \mathrm{C}^{45}$. Segundo recomendações contidas em protocolos clínicos, o tratamento da febre em pediatria deve ser restrito a utilização de antipirético em casos de temperatura muito elevada, motivo de desconforto para a criança ou quando tiver risco definido para uma determinada criança $\mathrm{a}^{11,37,47}$.

Mas também se postula que os clínicos têm conhecimentos deficientes sobre a febre e não assumem condutas diante de uma criança em estado febril. Tem-se demonstrado que os médicos, de modo geral, têm preocupações exageradas sobre a febre, da qual os leva a assumir condutas agressivas e de duvidosa utilidade para tratá-la, como são principalmente as combinações entre dois antipiréticos ${ }^{27}$. Se a febre representa resposta benéfica ou prejudicial à infecção tem sido motivo de debate durante muitos anos, mas as evidências indicam que é benéfica e sua supressão na maioria dos casos não tem benefício demonstrado ${ }^{48}$.

Assim, a falta de esclarecimento sobre os reais benefícios e riscos destes medicamentos, acrescido do fato de ser, a grande maioria de venda livre, tem levado ao seu uso indiscriminado, principalmente no meio pediátrico. Muitas vezes são prescritos sem um objetivo terapêutico específico, gerando iatrogenias e elevação do custo do tratamento, além de aumento de morbidade ${ }^{49-52}$.

Considerando o conceito de uso racional de medicamentos foi observado que em $40 \%$ das prescrições provenientes do SUS e em 64\% das N-SUS o medicamento não estava apropriado, ou seja, não estava adequado às características dos pacientes, aqui avaliado considerando somente faixa etária, e que não apresenta riscos de interação grave ou contraindicação. Seguindo o conceito de uso racional, quando se toma o percentual daqueles considerados adequados e verifica se a dose, a frequência e a duração do tratamento estão apropriadas, somente 3\% das prescrições do SUS e nenhuma do setor privado atendem ao critério de uso racional ou prescrição racional, não sendo diferentes estatisticamente.

\section{Considerações finais}

Esta pesquisa iniciou-se com indagações relacionadas às possíveis diferenças entre as prescrições pediátricas de AA e AINE provenientes do SUS e do setor privado, pois estudos desta natureza não foram encontrados. Pode-se concluir que na amostra avaliada não existem diferenças significantes entre as prescrições pediátricas contendo analgésicos, antipiréticos e anti-inflamatórios não esteroides. Os tratamentos estão focados somente em condutas medicamentosas descritas de maneira insuficiente, duvidosa, com uso diferentes daquelas recomendadas para faixa etária, frequência, dose e duração do tratamento, não constando nenhuma orientação não medicamentosa por escrito. Considerando o uso racional de medicamentos, esta análise mostrou que não exis- 
tem prescrições pediátricas de analgésicos, antipiréticos e anti-inflamatórios não esteroides que atendam a este critério no setor privado e, no SUS, não chegam a $3 \%$.

A educação e a informação desprovida de interesse comercial poderiam contribuir para o uso racional de medicamentos, além de medidas reguladoras mais severas quanto à prescrição pediátrica. Melhorar a qualidade da formação dos médicos, considerando o uso pediátrico de medicamentos antipiréticos, analgésicos e anti-inflamatórios não esteroides, reforçando programas acadêmicos, poderia ser uma das medidas preconizadas para melhoria do uso racional de medicamentos em crianças. Neste contexto, Instituições de ensino e profissionais de saúde deveriam assumir papeis transformadores.

\section{Colaboradores}

TR Ferreira participou da execução da parte experimental, coleta dos dados e redação do artigo; $\mathrm{AB}$ Ferreti da análise estatística e interpretação dos dados; S Barberato Filho da revisão crítica relevante do conteúdo intelectual; e LC Lopes da concepção do projeto, coordenação e correção final da redação. 


\section{Referências}

1. Sano PY, Masotti RR, Santos AAC, Cordeiro JA. Avaliação do nível de compreensão da prescrição pediátrica. J Pediatr 2002; 78(2):140-145.

2. Brasil. Lei $n^{\circ}$ 5.991, de 17 de dezembro de 1973. Dispõe sobre o controle sanitário do comércio de drogas, medicamentos, insumos farmacêuticos e correlatos, e dá outras providências. Diário Oficial da União 1973; 19 dez.

3. Brasil. Lei no 9.787, de 10 de fevereiro de 1999. Altera a Lei $n^{\circ} 6.360$, de 23 de setembro de 1976, que dispõe sobre a vigilância sanitária, estabelece o medicamento genérico, dispõe sobre a utilização de nomes genéricos em produtos farmacêuticos e dá outras providências. Diário Oficial da União 2000; $11 \mathrm{fev}$.

4. Brasil. Conselho Federal de Farmácia (CFF). Resolução no 357/01, de 20 de abril de 2001. Estabelece Regulamento Técnico das Boas Práticas de Farmácia. Diário Oficial da União 2001; 27 abr.

5. Brasil. Conselho Federal de Medicina (CFM). Resolução no 1.931 , de 17 de setembro de 2009. Código de ética médica. Diário Oficial da União 2009; 24 set.

6. Meiners MMMA, Bergsten-Mendes G. Prescrição de medicamentos para crianças hospitalizadas: como avaliar a qualidade? Rev Assoc Med Bras 2001; 47(4):332-337.

7. Abreu MM, Kowalski SC, Ciconelli RM, Ferraz MB. Apoios de decisão: instrumento de auxílio à medicina baseada em preferências. Uma revisão conceitual. Rev Bras Reumatol 2006; 46(4):266-272.

8. Agência Nacional de Vigilância Sanitária (Anvisa). Medicamentos. Registros de medicamentos. Como a Anvisa vê o uso off label de medicamentos. Brasília, 23 de maio de 2005. [página na Internet]. [acessado 2010 abr 27]. Disponível em: http://portal.anvisa. gov.br/wps/content/Anvisa+Portal/Anvisa/Inicio/Medicamentos/Assunto+de+Interesse/Medicamentos + novos/Como+a+Anvisa+ve+o+uso+off + label+ de+medicamentos

9. Arrais PSD, Barreto ML, Coelho HLL. Aspectos dos processos de prescrição e dispensação de medicamentos na percepção do paciente: estudo de base populacional em Fortaleza, Ceará, Brasil. Cad Saude Publica 2007; 23(4):927-937.

10. Bricks LF. Uso judicioso de medicamentos em crianças. J Pediatr (Rio J) 2003; 79(Supl.1):107-114.

11. Bricks LF, Silva CAA. Recomendações para o uso de anti-inflamatórios não hormonais em pediatria. Pediatria 2005; 27(2):114-125.

12. Klasco RK, editor. Drugdex System [Database on the Internet]. Greenwood Village (Colorado): Thomson Micromedex; 1974-2010. [acessado 2010 maio 4]. Disponível em: http://www.periodicos.caps.gov.br

13. Brasil. Ministério da Saúde (MS). Secretaria da Ciência, Tecnologia e Insumos Estratégicos. Departamento de Assistência Farmacêutica e Insumos Estratégicos. Formulário Terapeutico Nacional 2010. Brasilia. [página na Internet] 2010. [acessado 2013 set 27]. Disponível em: http://portal.saude.gov.br/ portal/arquivos/pdf/FTN.pdf
14. Fegadolli C, Mendes IJM, Simões MJS. Avaliação da prescrição médica em pediatria, baseada nos indicadores do uso de medicamentos selecionados pela OMS em Município do interior do Estado de São Paulo. Rev. Ciênc. Farm 2002; 23(2):239-254.

15. Oliveira GC. Relações Municipais de Medicamentos Essenciais no Estado de São Paulo: uma análise sob a ótica da essencialidade [dissertação]. Sorocaba: Universidade de Sorocaba; 2011.

16. British Medical Association and Royal Pharmaceutical Society of Great Britain. British National Formulary. $63^{\text {th }}$ Edition. London: BMJ Publishing Group, APS Publishing; 2012.

17. Simões MJS, Fegadolli C. Consumo de medicamentos por prescrição médica na assistência básica à saúde do município de Araraquara, SP. In: $1^{\circ}$ Seminário Brasileiro de Farmacoepidemiologia. Programas e resumos; 1996; Fortaleza. p.34.

18. Cunha MCN, Zorzatto JR, Castro LLC. Avaliação do uso de medicamentos na rede pública municipal de saúde de Campo Grande, MS. Revista Brasileira de Ciências Farmacêuticas 2002; 38(2):215-227.

19. Santos V, Nitrini SMO. Indicadores do uso de medicamentos prescritos e de assistência ao paciente de serviços de saúde. Rev Saude Publica 2004; 38(6):819-826.

20. Sanz EJ, Bergman U, Dahlström M. Paediatric drug prescribing: a comparison of Tenerife (Canary Islands, Spain) and Sweeden. Eur J Clin Pharmacol 1989; 37(1):65-68.

21. Bricks LF, Leone C. Use of medicines by children attending nurse's schools. Rev Saude Publica 1996; 30(6):527-535.

22. Haak H. Padrões de consumo de medicamentos em dois povoados da Bahia (Brasil). Rev Saude Publica 1989; 23(2):143-151.

23. Béria JU, Victora CG, Barros FC, Teixeira AB, Lombardi C. Epidemiologia do consumo de medicamentos em crianças de centro urbano da região sul do Brasil. Rev Saude Publica 1993; 27(2):95-104.

24. Wannmacher L. A ética do medicamento: múltiplos cenários. Uso Racional de Medicamentos: temas selecionados [periódico na internet]. jul 2007 [acessado 2008 dez 6]; 4(8):01-06. Disponível em: portal.saude.gov.br/portal/arquivos/pdf/v4n 8 _ etica_medicamentos.pdf

25. Laporte JR, Tognoni G, Rozenfeld S. Epidemiologia do medicamento: princípios gerais. São Paulo: $\mathrm{Hu}-$ chee-Abrasco; 1989.

26. Melamud A, Suwzda A, Matamoros R, Ringuelet L. Indicación de antitérmicos por médicos pediatras: Internet como modalidad de recolección de datos. Arch Argent Pediatr 2008; 106(5):404-408.

27. Saad MD, Galagarra DP, Alcalá NA, Nino EC, Talavera LM. Conocimientos sobre fiebre y conductas de los residentes del postgrado de pediatría ante un paciente febril. Arch Venez Pueric Pediatr 2006; 69(3):96-102.

28. Simões MJS, Fegadolli C. Antibiotic treatmente in daily pratice for children seen at public health units. Pharmacoepidemiol Drug Saf 1999; 8(2):122-123. 
29. Ribeiro AQ, Sevalho G, César CC. Utilização prévia de anti-inflamatórios não esteróides por pacientes encaminhados para endoscopia em um hospital universitário brasileiro. Rev. Ciênc. Farm. Básica Apl 2007; 28(1):67-75.

30. Bricks LF. Analgésicos antitérmicos e anti-inflamatórios não hormonais: controvérsias sobre sua utilização em crianças. Pediatria (São Paulo) 1998; 20(3):230-246.

31. Bricks LF, Sih T. Medicamentos controversos em otorrinolaringologia. J Pediatr 1999; 75(1):11-22.

32. Silva $\mathrm{CH}$, Giugliani EJ. Consumo de medicamentos em adolescentes escolares: uma preocupação. J Pediatr 2004; 80(4):326-332.

33. Moraes CG, Tavares NUL, Mengue SS, Dal Pizzol TS. Utilização de medicamentos entre crianças de zero a seis anos: um estudo de base populacional no sul do Brasil. Cien Saude Colet 2013; 18(12):35833593.

34. Gosalakkal JA, Kamoji V. Reye syndrome and reyelike syndrome. Pediatr Neurol 2008; 39(3):198-200.

35. Schrör K. Aspirin and Reye syndrome: a review of the evidence. Paediatr Drugs 2007; 9(3):195-204.

36. Glasgow JF, Middleton B, Moore R, Gray A, Hill J. The mechanism of inhibition of beta-oxidation by aspirin metabolites in skin fibroblasts from Reye's syndrome patients and controls. Biochim Biophys Acta 1999; 1454(1):115-125.

37. Brook I. Unexplained fever in young children: how to manage severe bacterial infections. BMJ 2003; 327(7423):1094-1097.

38. Lesko SM, Mitchell AA. The safety of acetaminophen and ibuprofen among children younger that two years old. Pediatrics 1999, 104(4):e39.

39. Litalien C, Jacqz-Aigrain E. Risks and benefits of nonsteroidal anti-inflammatory drugs in children: a comparison with paracetamol. Paediatr Drugs 2001; 3(11):817-858.

40. Maj S, Centkowski P. A study of the incidence of agranulocytosis and aplastic anemia associated with the oral use of metamizole sodium in Poland. Med Sci Monit 2004; 10(9):PI93-PI95.

41. Backstrom M, Hauml S, Mjorndal T, Dahlqvist R. Utilization pattern of metamizole in northern Sweden and risk estimates of agranulocytosis. Pharmacoepidemiol Drug Saf 2002; 11(3):239-245.

42. Hedenmalm K, Spigset O. Agranulocytosis and other blood dyscrasias associated with dipyrone (metamizole). Eur J Clin Pharmacol 2002; 58(4):265274.
43. Ibanez L, Vidal X, Ballarin E, Laporte JR. Agranulocytosis associated with dipyrone (metamizol). Eur J Clin Pharmacol 2005; 60(11):821-829.

44. Edwards JE, Meseguer F, Faura CC, Moore RA, McQuay HJ. Single dose dipyrone for acute postoperative pain. Cochrane Database of Systematic Reviews 2001; (3):CD003227.

45. Moraga F, Horwitx A, Romero C. Fever phobia parents knowledge and attitude towards fever. Rev Chil Pediatr 2007; 78(2):160-164.

46. Rati RMS, Goulart LMHF, Alvim CG, Mota JAC. "Criança não pode esperar": A busca de serviço de urgência e emergência por mães e suas crianças em condições não urgentes. Cien Saude Colet 2013; 18(11):3663-3672.

47. Avner JR, Baker MD. Management of fever in infants and children. Emerg Med Clin North Am 2002; 20(1):49-67.

48. Betz MG, Grunfeld AF. "Fever phobia" in the emergency department: a survey of children's caregivers. Eur J Emerg Med 2006; 13(3):129-133

49. Wannmacher L, Ferreira MBC. Febre: mitos que determinam condutas. Brasília: Uso Racional de Medicamentos: Temas Selecionados; 2004. 1(9). [página na Internet]. [acessado 2013 set 27]. Disponível em: http://portal.saude.gov.br/portal/arquivos/pdf/Febre.pdf

50. Bricks LF. Uso de anti-inflamatórios não hormonais em crianças com doenças virais vs. risco potencial de síndrome de Reye e doenças invasivas graves pelo Streptococcus do grupo A. Pediatria 2000; 22:35-43.

51. American Academy of Pediatrics. Acetaminophen toxicity in children. Pediatrics 2001; 108(4):102-104.

52. Berde CB, Sethna NF. Drug therapy: analgesics for the treatment of pain in children. N Engl J Med 2002; 347(14):1094-1103.

Artigo apresentado em 29/09/2012

Aprovado em 02/10/2012

Versão final apresentada em 04/10/2012 